\title{
Serum Adenosine Deaminase Level is High But Not Related with Disease Activity Parameters in Patients with Rheumatoid Arthritis
}

\author{
Gülseren Demir ${ }^{1}$, Pınar Borman ${ }^{*}, 2$, Figen Ayhan ${ }^{1}$, Tuba Özgün ${ }^{3}$, Ferda Kaygısız ${ }^{1}$ and Gulsen Yilmez ${ }^{3}$ \\ ${ }^{1}$ Ankara Training and Research Hospital, Clinic of Physical Medicine and Rehabilitation, ${ }^{2}$ University of Hacettepe, \\ Faculty of Medicine, Dept of Physical Medicine and Rehabilitation, ${ }^{3}$ Ankara Training and Research Hospital, Clinic of \\ Biochemistry, Turkey
}

\begin{abstract}
Serum adenosine deaminase (ADA) has been previously proposed to predict disease activity in patients with rheumatoid arthritis (RA). The aim of this study was to investigate the level of serum ADA, and the relationship between ADA and disease activity markers, in a group of patients with RA.

A hundred and 10 patients with a diagnosis of RA were recruited from outpatient clinic of Rheumatology Unit. Demographic properties comprising age, gender, disease duration and drugs were recorded. Disease activity based on disease activity score (DAS)28-erythrocyte sedimentation rate (ESR) and DAS28- C reactive protein (CRP,) ESR, CRP levels, as well as pain by visual analog scale and rheumatoid factor (RF) were recorded. Serum ADA levels (IU/L) were determined in all RA patients and in 55 age and sex similar healthy control subjects.

Ninety-six female and 14 male RA patients with a mean age of $54.32 \pm 11.51$, and with a mean disease duration of $11.5 \pm 9.13$ years were included to the study. The control group comprised of 48 female and 7 male healthy subjects. $35.5 \%$ of the patients were on methotrexate (MTX) and $64.5 \%$ of patients were on combined DMARDs or combined MTX and anti-TNF therapies. The mean serum ADA level was statistically higher in RA patients than in control subjects $(27.01 \pm 10.6 \mathrm{IU} / \mathrm{L} v s 21.8 \pm 9.9 \mathrm{IU} / \mathrm{L})$. The mean values of ESR $(23.2 \pm 14.8 \mathrm{~mm} / \mathrm{h}), \mathrm{CRP}(1.71 \pm 1.11 \mathrm{mg} / \mathrm{dL})$, pain by VAS (37.2 \pm 27.1$)$, DAS28-ESR (2.72 \pm 0.77$)$, DAS28 CRP (1.37 \pm 0.5$)$ were not correlated with ADA levels ( $>0.05)$.

Our results have shown that serum ADA levels are higher in RA patients than in controls but were not related with any of the disease activity markers. We conclude that ADA in the serum may not be a reliable biochemical marker to predict disease activity in patients with RA.
\end{abstract}

Keywords: Adenosine deaminase, disease activity, rheumatoid arthritis, serum.

\section{INTRODUCTION}

Rheumatoid Arthritis (RA) is a chronic inflammatory multisystem disorder affecting primarily cartilage and joints, which aetiopathogenesis has not been clarified yet. The synovial inflammation and destruction of joints is often progressive and lead to joint destruction $[1,2]$. As a result of cell-mediated immune response, the condition is characterized by secretion of different inflammatory products from activated lymphocytes, macrophages, fibroblasts and leukocytes in synovial joints. Endogenous adenosine suppress this process and actually adenosine pathway mediates the inflammatory cascade [2, 3]. Adenosine indicates its modulator effects by interaction with $\mathrm{G}$ protein-coupled receptors, that exists in at least three molecular forms; A1, A2A, A2B and A3. In this regard, extracellular adenosine levels are important in determining its ability of regulating various biological processes $[4,5]$. Adenosine is metabolized by adenosine deaminase (ADA);

*Address correspondence to this author at the University of Hacettepe, Faculty of Medicine, Department of Physical Medicine and Rehabilitation, Sihhiye, Ankara, Turkey; Tel: 90.532.4649897; Fax: 90.312.4186363;

E-mail: pinarborman@gmail.com one of the main enzymes of purine metabolic pathway. This catabolic enzyme ADA represents an exclusive checkpoint in the regulation of extracellular adenosine levels and, consequently, in the control of immune system activity through its modulation of adenosine pathways in several conditions like RA [4-14]. Therefore determination of serum adenosine level can be an appropriate way to assess disease activity in RA.

There are conflicting reports about the relationship of ADA and disease activity parameters in the English literature [5-16]. Serum ADA was reported to be increased in serum and synovial fluid of RA patients compared with noninflammatory arthritic conditions $[7,10,11]$. ADA has been suggested as a prediction marker or a useful biochemical marker of inflammatory process in patients with RA [13, 14], while some studies did not find a correlation between ADA levels and disease activity markers $[15,16]$. In addition most of these studies have been performed with small sample size and have not explained patient characteristics and drugs in details [6-16].

The aim of this study was to determine the level of ADA in a large group of RA patients and evaluate its association with disease activity parameters. 


\section{MATERIALS AND METHODS}

A hundred and ten patients with RA, who met the American College of Rheumatology revised criteria [17] for RA, were enrolled from the outpatient clinics of Ankara Training and Research Hospital, Rheumatology unit of Physical Medicine and Rehabilitation Clinic, between 2012 January and December. Patients with a mean BMI $>35$, aged $<18$, and $>80$ years patients with these comorbid diseases (chronic renal failure, hepatic insufficiency, thyroid disease, heart failure, tuberculosis, septic conditions and uncontrolled diabetes mellitus) were not included to the study. 144 patients with rheumatoid arthritis were assessed for eligibility. 5 patients refused to participate in the study and 29 patients were excluded due to age or comorbid diseases. Therefore $110 \mathrm{RA}$ patients were included to the study. Fifty- five age and sex similar (within \pm 2 years) healthy control subjects who were the members of the hospital staff and volunteered to participate in the study, were comprised. We obtained informed consent from all the patients and controls, and the study was approved by the ethics committee of the hospital.

Demographic characteristics including age, sex, body mass index (BMI) in all subjects, and drug intake and disease duration of RA patients were recorded. All patients underwent a clinical interview and physical examination. In order to determine disease activity in patients with RA, disease activity score (DAS)28 was performed [18].

Blood samples of the patients were taken to determine hemoglobin $(\mathrm{Hb})$, erythrocyte sedimentation rate (ESR) using the Westergren method, rheumatoid factor (RF) using nephelometric method and $\mathrm{C}$ reactive protein (CRP) by turbidimetric method in RA group. The numbers of tender and swollen joints as well as joint pain assessed by visual analog scale (VAS) were collected from patient files. DAS 28-ESR and DAS28-CRP were calculated for each patient using well-known calculations which included the number of tender and swollen joints, ESR and CRP, and patients' global assessment of general health expressed on visual analog scale [18]. The ESR, CRP, DAS28 and pain by VAS were considered as disease activity parameters.

Serum ADA levels in patient and control groups were determined and the relation between ADA level and disease activity parameters were evaluated. Although the exact effect of MTX on the ADA enzymatic activity is not clearly understood, it is assumed that in purine enzyme metabolic pathway, MTX increases adenosine and inhibits ADA both directly and indirectly [10]. Therefore the RA group was divided into two groups as patients receiving MTX and patients on drugs other than MTX. Subgroup analysis was performed in regard to ADA and related activity parameters.

The serum level of ADA was determined in all subjects. Serum samples were obtained from venous blood, routine laboratory investigations were performed on samples of RA patients and after this all serum samples were frozen immediately. The samples were stored at $-80^{\circ} \mathrm{C}$ until measuring time. ADA activity was determined by an enzymatic spectrophotometric method with Schimadzu CL770 clinical spectrophotometer [19].

\section{Statistical Analysis}

Data analysis was performed using the SPSS statistical package program version 15.0 for Windows. Data for variables were described using mean $\pm \mathrm{SD}$. The comparisons for continuous variables between the two groups (patients and controls) were performed by independent sample $t$ test and Mann-Whitney U test as appropriate.

Correlation analysis for continuous variables was performed using the Spearman's correlation test. Scatter plot was performed to indicate the correlation between two continuous variables (ADA and DAS28). The value of $\mathrm{p}<0.05$ was considered significant.

\section{RESULTS}

The study group comprised 110 RA patients and 55 healthy control subjects. The demographic characteristics of the subjects are shown in Table 1. No statistical difference was observed between the groups according to age, sex and BMI $(p>0.05)$. The clinical features including the disease activity parameters of RA patients are shown in Table $\mathbf{2}$. Thirty-two $(35.5 \%)$ of the patients were on methotrexate (MTX) and $64.5 \%$ of patients were on combined disease modifying antirheumatic drug (DMARD)s or combined MTX and anti- tumor necrosis factor (TNF) therapies, 14 patients $(12 \%)$ were on leflunomide (LEF) therapy, 44 patients $(40 \%)$ were using corticosteroids and/or NSAIDs in addition to DMARDs. All patients using MTX were also receiving folic acid supplementation $5 \mathrm{mg} /$ week. The mean MTX and folic acid dosages were $15.4 \mathrm{mg} /$ week and 5 $\mathrm{mg} /$ week respectively. The mean ADA level was determined as $27.01 \pm 10.6 \mathrm{IU} / \mathrm{L}$ in the RA group and was found to be statistically higher than in the control group $(21.8 \pm 9.9 \mathrm{IU} / \mathrm{L})$, (independent sample $\mathrm{t}$ test, $\mathrm{p}<0.05$ ). No relationship was observed between the mean ADA level and disease activity parameters (Spearman correlation test, $\mathrm{p}>0.05$ ) (Figs. 1, 2).

Table 1. The demographic characteristics of the patients and control subjects (descriptives).

\begin{tabular}{|c|c|c|}
\hline & RA (n=110) & Control (n=55) \\
\hline \hline Age $($ mean \pm SD) years & $54.32 \pm 11.51$ & $49.8 \pm 9.83$ \\
\hline Sex $(\mathrm{F} / \mathrm{M})$ & $97 / 13$ & $45 / 10$ \\
\hline Body mass index $\left(\mathrm{kg} / \mathrm{m}^{2}\right)$ & $25.8 \pm 5.4$ & $26.9 \pm 7.8$ \\
\hline Disease duration (years) & $11.5 \pm 9.13$ & - \\
\hline
\end{tabular}

RA: Rheumatoid arthritis.

Depending on the assumption that MTX decreases the inflammation by stimulating the adenosine receptors and by inhibiting ADA, we assessed the serum ADA of RA patients receiving MTX and taking DMARDs other than MTX. Patients with RA were divided into two groups according to the use of MTX. According to this classification, 71 patients were on MTX while 39 were on DMARDs other than MTX. There was no statistically significant difference between patients with MTX and patients with other DMARDs, in regard to serum ADA level (independent sample t test, $\mathrm{p}>0.05,27.53 \pm 11.4$ vs $24.16 \pm 7.8$ respectively). we evaluated the relationship between disease activity markers and ADA 
levels in groups with regard to MTX usage, and we could not find any relationship between disease activity markers and ADA levels in none of these groups (Table 3, Spearman correlation analysis, $\mathrm{p}>0.05$ ).

Table 2. The mean \pm SD values of the clinical activity parameters and RF levels in the patient group (descriptives).

\begin{tabular}{|c|c|}
\hline & $\begin{array}{c}\text { RA (n=110) } \\
\text { Mean } \pm \text { SD (Min-Max) }\end{array}$ \\
\hline \hline Pain by VAS & $27.22 \pm 27.17(0-100)$ \\
\hline ESR $(\mathrm{mm} / \mathrm{h})$ & $23.28 \pm 14.89(2-65)$ \\
\hline CRP $(\mathrm{mg} / \mathrm{dL})$ & $1.15 \pm 0.6(0.3-14.4)$ \\
\hline DAS28-ESR & $2.72 \pm 0.78(0.68-4.47)$ \\
\hline DAS28-CRP & $1.37 \pm 0.51(0.96-2.82)$ \\
\hline RF & $122.01 \pm 156.59(10-640)$ \\
\hline
\end{tabular}

VAS: visual analog scale, ESR: erythrocyte sedimentation rate, CRP:C-reactive protein, DAS: Disease activity score, RF: Rheumatoid factor, min: minimum, max: maximum, SD: standard deviation.

\section{DISCUSSION}

Serum ADA was previously reported to be increased in serum and synovial fluid of RA patients compared with noninflammatory arthritic conditions [7, 10-12, 20]. Yuksel et al. found an increased level of ADA activity in synovial fluid of patients with RA, than in their control group [11].
Zekeri et al. indicated higher serum ADA activity in RA patients when compared with osteoarthritic patients [12]. Most of the previous studies had shown a significant relation between the mean serum ADA level and disease activity markers [7, 11, 13, 14] Sari et al. investigated the relation betweeen serum ADA level and its isoenzymes with disease activity of RA and showed that total ADA and ADA2 had correlation with RA activity [7]. Nalesnik et al. have shown that ADA activity is related with inflammation and serum ADA could be a useful biochemical marker of inflammatory process in RA [14]. Zamani et al. determined a relationship between ADA and disease activity and reported that 'serum ADA may help to predict disease activity in patients with RA' [13]. On the other hand Erer et al. could not find a relation between ADA levels and disease activity parameters in their RA group receiving DMARD and/or anti TNF therapy [15]. Also Cordero did not define a relation between serum ADA and disease activity in their study group [16]. In this study we determined higher levels of ADA in RA patients than in healthy control subjects similar to previous data, but could not find a significant relationship between ADA activity and disease activity parameters. In our group, disease activity was relatively not so high compared to previous papers, which might be related with the maintenance of variable treatments during the study. We speculate that the ADA levels might be related with disease activity parameters if the study group included patients with high disease activity and without treatment. As this is not ethical, further studies with recently diagnosed RA patients may clarify these points and would be of importance.

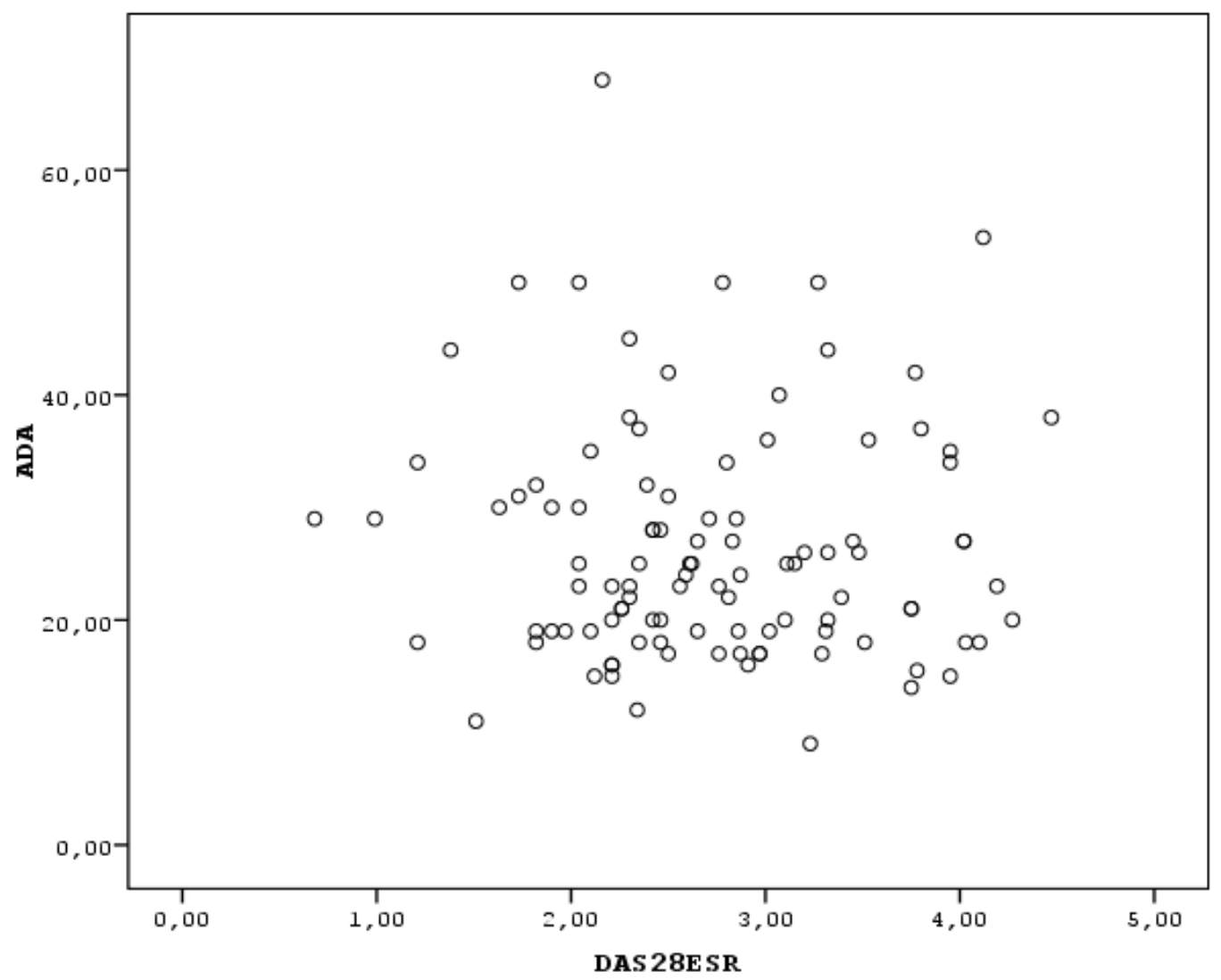

Fig. (1). The correlation between of ADA levels and DAS28-ESR values, indicated as scatter plot. 


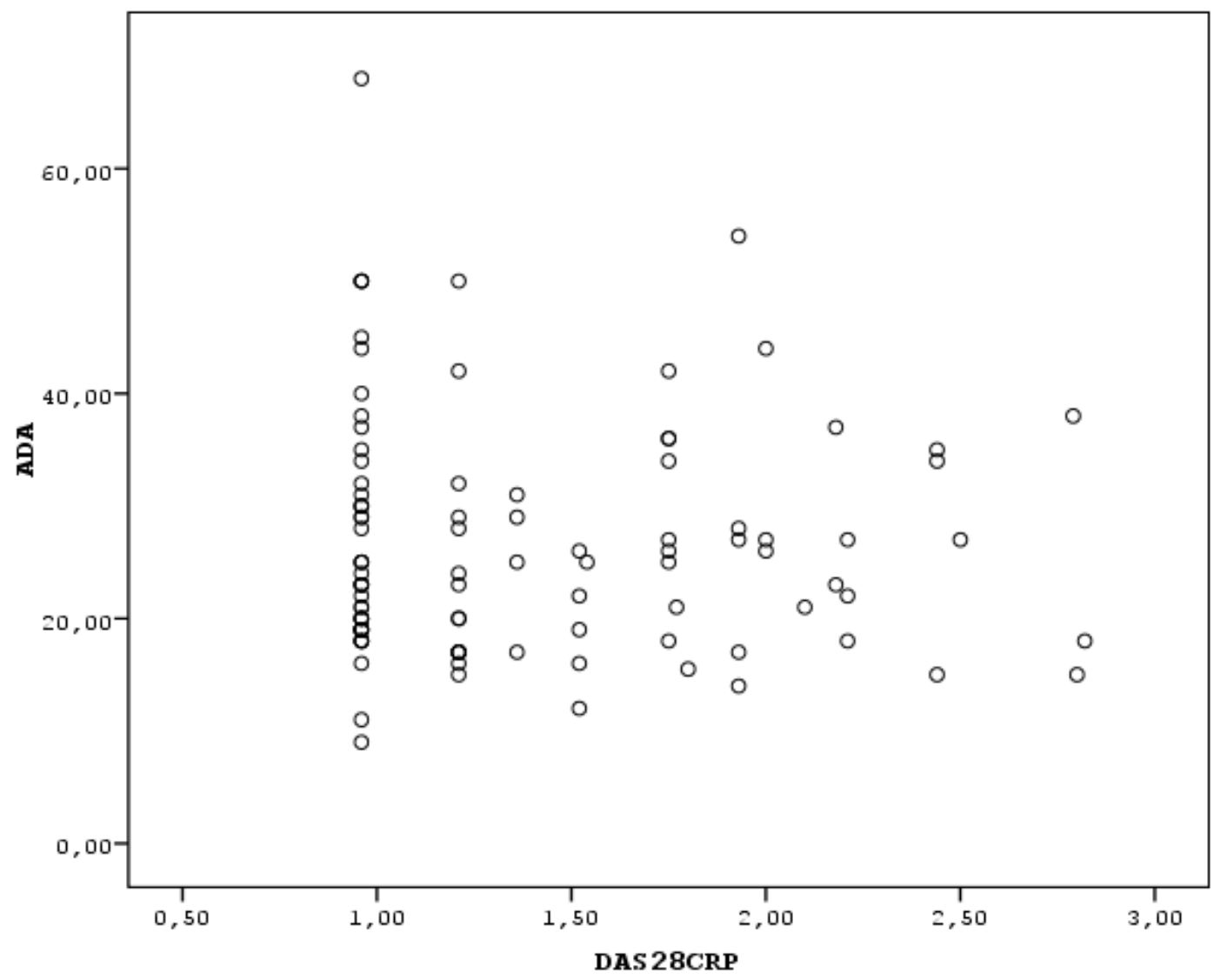

Fig. (2). The correlation between ADA levels and DAS28-CRP values indicated as scatter plot.

Table 3. The relationship between ADA and disease activity parameters in both patient groups using MTX and using other DMARDs (Spearman correlation test, p>0.05).

\begin{tabular}{|c|c|c|}
\hline & $\begin{array}{c}\text { ADA Patients on MTX } \\
(\mathbf{n}=\mathbf{7 1})\end{array}$ & $\begin{array}{c}\text { ADA Patients on Other } \\
\text { DMARD (n=39) }\end{array}$ \\
\hline \hline ESR & $\mathrm{p}=0.370$ & $\mathrm{p}=0.221$ \\
& $\mathrm{r}=0.111$ & $\mathrm{r}=0.209$ \\
\hline CRP & $\mathrm{p}=0.475$ & $\mathrm{p}=0.592$ \\
& $\mathrm{r}=0.088$ & $\mathrm{r}=0.092$ \\
\hline DAS28-ESR & $\mathrm{p}=0.736$ & $\mathrm{p}=0.535$ \\
& $\mathrm{r}=0.042$ & $\mathrm{r}=-0.107$ \\
\hline DAS28-CRP & $\mathrm{p}=0.839$ & $\mathrm{p}=0.491$ \\
& $\mathrm{r}=-0.025$ & $\mathrm{r}=0.119$ \\
\hline Pain by VAS & $\mathrm{p}=0.781$ & $\mathrm{p}=0.606$ \\
& $\mathrm{r}=0.034$ & $\mathrm{r}=0.089$ \\
\hline
\end{tabular}

ADA: Adenosine deaminase, MTX: methotrexate, DMARD: Disease modifying antirheumatic drug, VAS: visual analog scale, ESR: erythrocyte sedimentation rate, CRP:C-reactive protein, DAS: Disease activity score.

Serum ADA reflects monocyte/macrophage activity in several inflammatory disease like RA, viral conditions or tuberculosis $[8,9,21]$. Etiology of RA is considered mostly to be a $\mathrm{T}$ helper- mediated disease. Endogenous adenosine has anti-inflammatory function but due to some local factors, its concentration may decrease in the emission area. Inflammation triggers adenosine release suppression and elevation of ADA activity, which causes adenosine disturbance and lead to decreased enzyme activity which disintegrates ATP to adenosine. Therefore reduction of local concentration of adenosine by ADA may contribute to joint inflammation of RA [5, 10, 22, 23]. Regarding these points; the higher levels of ADA found in our and previous RA patients are not surprising.

Methotrexate is the anchor drug for therapy of RA and more often its combination with other DMARDs is used commonly. Since the anti-inflammatory effect of MTX is via the stimulation of adenosine receptors, it was considered that the therapeutic effects of MTX could be as a result of catalytic activity of ADA in the serum [5, 10]. The pathophysiology of adenosine secretion is also related with MTX pathways [10,24-26]. Previous studies indicated that 'adenosine is an effector molecule mediating the therapeutic effects of MTX via adenosine receptor signalling' [5, 14]. It is suggested that MTX inhibits ADA and increases vasodilatation induced by adenosine. Therefore it is an assumption that ADA activity in serum may be a possible biochemical marker for monitoring the therapeutic effects of MTX in RA patients [5, 14]. Nalenisk et al. determined a significant correlation between ADA and CRP concentrations in serum of RA patients who were not on MTX therapy [14]. Van Ede et al. concluded a relationship between ADA and MTX treatment in patients receiving MTX and folic acid [26, 27]. Salesi et al. determined ADA levels before and after the MTX treatment in their RA group and indicated an association with ADA levels with the efficacy of MTX treatment [10]. But these studies did not 
defined disease activity status nor the MTX and/or folic acid dosages of their patients. In our study we have determined ADA levels with regard to MTX usage but could not find a difference between the groups. In addition we could not detect a relationship between ADA level and disease activity in patients with and without MTX treatment. The mean dosage of MTX was $15.6 \mathrm{mg}$ and disease activity was relatively not so high, in our study group. The different results of our data from the previous literature may be due to MTX and/or folic acid dosages or disease activity status.

There may be some limitations of our study. One of them is the absence of detection of ADA isoenzymes. Also not covering the synovial ADA levels to compare and determine the relationship with activity markers may be accepted as another limitation. The major strengths of the present study include a large sample of RA patients, defining the therapies in details and inclusion of the control group.

In conclusion this study showed that level of ADA was higher in Turkish RA patients than in healthy control subjects but not related with any of the disease activity parameters. We suggest that ADA activity does not seem to help to predict disease activity. According to our results traditional disease activity parameters still remain the best strategy for determining the activity and monitoring the treatment in patients suffering from RA.

\section{CONFLICT OF INTEREST}

The authors confirm that this article content has no conflict of interest.

\section{ACKNOWLEDGEMENTS}

Declared none.

\section{REFERENCES}

[1] Brasington RD. Clinical features of rheumatoid arthritis. In: Hochberg MC, Silman AJ, Smolen JS, Eds. Rheumatology. $5^{\text {th }}$ ed. Philadelphia: Mosby-Elsevier 2011; pp. 829-38.

[2] Mc Innes IB, Schett G. The pathogenesis of rheumatoid arthritis. N Engl J Med 2011; 365: 2205-9.

[3] Iwaki-Egawa S, Yamamoto T, Watanabe Y. Human plasma adenosine deaminase2 is secreted by activated monocytes. Biol Chem 2006; 387: 319-21

[4] Nakamachi Y, Koshiba M, Nakazawa T, et al. Specific increase in enzymatic activity of adenosine deaminase 1 in rheumatoid synovial fibroblasts. Arthritis Rheum 2003; 48: 668-74.

[5] Antonioli L, Colucci R, La Motta C, et al. Adenosine deaminase in the modulation of immune system and its potential as a novel target for treatment of inflammatory disorders. Curr Drug Targets 2012;13(6):842-62.

[6] Hitoglue S, Hatzstilianaou M, Gougoustamaou D, Athanassiadou F, Kotsis A, Catriu D. Adenosine deaminase activity and its isoenzyme pattern in patients with juvenile rheumatoid arthritis and systemic lupus erythematosus. Clin Rheumatol 2001; 20: 411-6.

[7] Sari RA, Taysi S, Yilmaz O, Bakan N. Correlation of serum levels of adenosine deaminase activity and its isoenzymes with disease activity in rheumatoid arthritis. Clin Exp Rheumatol 2003; 21: 87-90.

[8] Calıs M, Ates F, Yazıcı C, et al. Adenosine deaminase enzyme levels, their relation with disease activity, and the effect of colchicine on adenosine deaminase levels in patients with Behcet's disease. Rheumatol Int 2005; 25: 452-6.

[9] Meunier P, Filipe P, Emerit I Freitas J, Guerra Rodrigo F, Manso C. Adenosine deaminase in progressive systemic sclerosis. Acta Derm Venerol 1995; 75: 297-9.

[10] Salesi M, Aghaye-Ghazvini R, Farajzadegan Z, et al. Serum adenosine deaminase in patients with rheumatoid arthritis treated with methotrexate. J Res Pharm Pract 2012; 1(2): 72-6.

[11] Yuksel H, Akoğlu TF. Serum and synovial fluid adenosine deaminase activity in patients with rheumatoid arthritis, osteoarthritis and reactive arthritis. Ann Rheum Dis 1988; 47: 4925 .

[12] Zekeri Z, Izadi S, Niazi A, et al. Comparison of adenosine deaminase levels in serum and synovial fluid between patients with rheumatoid arthritis and osteoarthritis. Int J Clin Exp Med 2012; 5(2): 195-200.

[13] Zamani B, Jamali R, Jamali A. Serum adenosine deaminase may predict disease activity in rheumatoid arthritis. Rheumatol Int 2012; 32: 1967-75.

[14] Nalesnik M, Nikolic JM, Jandric S. Adenosine deaminase and Creactive protein in diagnosing and monitoring of rheumatoid arthritis. Med Glas (Zenica) 2011; 8(1): 163-8.

[15] Erer B, Yilmaz G, Yilmaz FM, Koklu S. Assessment of adenosine deaminase levels in rheumatoid arthritis patients receiving antiTNF $-\alpha$ therapy. Rheumatol Int 2009; 29: 651-4.

[16] Cordero OJ, Salgado FJ, Mera-Varela A, Nougueira M. Serum interleukin-12, interleukin-15, soluble CD26, and adenosine deaminase in patients with rheumatoid arthritis. Rheumatol Int 2001; 21(2): 69-74.

[17] Arnett FC, Edworthy SM, Block DA, et al. American College of Rheumatology 1987 revised criteria for the classification of rheumatoid arthritis. Arthritis Rheum 1988, 31: 315-24.

[18] Wells G, Becker JC, Teng J, et al. Validation of the 28-joint disease activity scoe (DAS28) and EULAR response criteria based on CRP against disease progression in patients with rheumatoid arthritis, and comparison with the DAS28 based on erythrocyte sedimentation rate. Ann Rheum Dis 2009; 68: 954-60.

[19] Ungerer JP, Oosthuizen HM, Bissbort SH, Vermaak JH. Serum adenosine deaminase: Isoenzymes and diagnostic application. Clin Chem 1992; 38(7): 1322-6.

[20] Pallinti V, Ganesan N, Anbazhagan M, Rajasekhar G. Serum biochemical markers in rheumatoid arthritis. Indian $\mathrm{J}$ Biochem Biophys 2009; 46: 342-4.

[21] Tuon FF, Litvoc MN, Lopes MI. Adenosine deaminase and tuberculosis pericarditis- a systematic review with meta-analysis Acta Trop 2006; 99: 67-74.

[22] Nakamachi Y, Koshiba M, Nakazawa T, et al. Specific increase in enzymatic activity of adenosine deaminase 1 in rheumatoid synovial fibroblasts. Arthritis Rheum 2003; 48: 668-74.

[23] Cronstein BN, Naime D, Firestein G. The anti-inflammatory effects of an adenosine kinase inhibitor are mediated by adenosine. Arthritis Rheum 1995; 38(8): 1040-5.

[24] Riksen NP, Barrera P, Van den Broek PHH, van Riel PLCM, Smits $P$. Methotrexate modulates the kinetics of adenosine in humans in vivo. Ann Rheum Dis 2006; 65: 465-70.

[25] Cronstein BN. Going with flow: methotrexate, adenosine and blood flow. Ann Rheum Dis 2006; 65: 421-2.

[26] Van Ede AE, Laan RFJM, De Abreu RA, Stegeman ABJ, van de Putte LBA. Purine enzymes in patients with rheumatoid arthritis treated with methotrexate. Ann Rheum Dis 2002; 61: 1060-4.

[27] Baggott JE, Morgan ST, Ha TS, Alarcon GS, Koopman WJ, Krumdieck CL. Antifolates in rheumatoid arthritis: a hypothetical mechanism of action. Clin Exp Rheumatol 1993; 11(Suppl 8): S101-5. 